\title{
Prediction of longitudinal dispersion coefficient using multivariate adaptive regression splines
}

\author{
Amir Hamzeh Haghiabi \\ Water Engineering Department, Lorestan University, Khorramabad 0098-663-4200289, Iran. \\ e-mail: ahaghiabi@gmail.com
}

In this paper, multivariate adaptive regression splines (MARS) was developed as a novel soft-computing technique for predicting longitudinal dispersion coefficient $\left(D_{L}\right)$ in rivers. As mentioned in the literature, experimental dataset related to $D_{L}$ was collected and used for preparing MARS model. Results of MARS model were compared with multi-layer neural network model and empirical formulas. To define the most effective parameters on $D_{L}$, the Gamma test was used. Performance of MARS model was assessed by calculation of standard error indices. Error indices showed that MARS model has suitable performance and is more accurate compared to multi-layer neural network model and empirical formulas. Results of the Gamma test and MARS model showed that flow depth $(H)$ and ratio of the mean velocity to shear velocity $\left(u / u^{*}\right)$ were the most effective parameters on the $D_{L}$.

\section{Introduction}

Assessment of river water quality is a major factor for developing environmental projects. Therefore, governments construct sampling stations along the rivers to continuously monitor river water quality. Unfortunately, sometimes, rivers are considered as a place for sewage disposal that causes pollution. Therefore, study of mixing flow to reduce degree of contamination has become one of the main subjects in river water quality projects (Leta et al. 2014). When a source of pollution, which has a density close to density of water, is injected into the river, it spreads in the river and is transmitted downstream due to molecular motion and turbulence of flow. Studies on river water quality have focused on the mechanism of mixing flow to reduce the effect of pollution on public environmental health. Dispersion coefficient is the main parameter which is discussed in river water quality problems (Ji 2008; Benedini and Tsakiris 2013). At the initial stage of injection of pollution, dispersion mechanism is three-dimensional whereas with widespread pollution in the flow section and moveling, the pollution along the river dispersion mechanism assumes to be one-dimensional (figure 1). As seen in figure 1, in stage $(\mathrm{V})$, dispersion is almost one-dimensional.

Several experimental studies have been conducted on dispersion coefficient in rivers. Most of these studies are based on routing tracer concentration along the river (Atkinson and Davis 2000; Davis and Atkinson 2000; Davis et al. 2000; Disley et al. 2015; Parsaie and Haghiabi 2015a, b, c, d). Due to the high cost of experimental studies, investigators have attempted to implement mathematical methods for modelling $D_{L}$. In mathematical approaches, the governing equations have been solved by powerful methods such as finite difference, finite volume and finite element methods (Stamou 1992; Wang et al. 2015). The advection-dispersion equation (ADE) is the governing equation on one-dimensional pollution transmission in rivers.

Keywords. River water quality; artificial neural network; longitudinal dispersion coefficient; pollution transmission. 


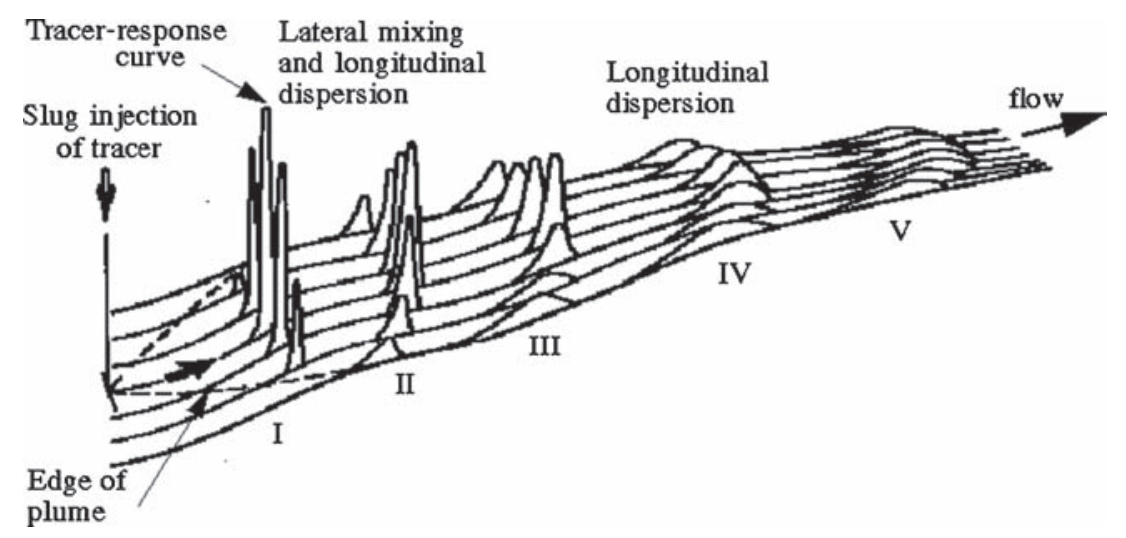

Figure 1. Schematic map of dispersion mechanism in rivers (Benedini and Tsakiris 2013).

Equation (1) presents one-dimensional ADE without any since or source terms (Chao et al. 2007).

$$
\frac{\partial C}{\partial t}+u \frac{\partial C}{\partial x}=D_{L} \frac{\partial^{2} C}{\partial x^{2}}
$$

where $C$ is the concentration of pollution, $D_{L}$ is the longitudinal dispersion coefficient, $u$ is the mean velocity of river flow. El Kadi Abderrezzak et al. (2015) and Parsaie and Haghiabi (2015a, b, c, d) investigated the role of $D_{L}$ in numerical modelling of pollution transmission in rivers. They stated that accurate prediction of $D_{L}$ has significant effect on numerical simulation; therefore, investigators have used powerful approaches, especially softcomputing techniques, for predicting this parameter. In this regard, the use of artificial neural network (ANN) (Kashefipour and Falconer 2002; Noori et al. 2015a), radial basis function neural network (RBF) (Parsaie and Haghiabi 2015a, b, c, d), genetic programming (gp) (Azamathulla and Ghani 2011), support vector machine (SVM) (Azamathulla and Wu 2011), adaptive neuro-fuzzy inference system (ANFIS) (Riahi-Madvar et al. 2009; Noori et al. 2015a), group method of data handling (GMDH) (Najafzadeh and Sattar 2015), and gene expression programing (GEP) (Sattar and Gharabaghi 2015) have been reported. Based on the reports, accuracy of soft-computing methods is high compared to empirical formulas. Parsaie and Haghiabi (2015a, b, c, d) proposed a novel approach for numerical simulation of pollution transmission in rivers based on using ANN model together with finite volume method (FVM). They used ANN to estimate $D_{L}$ and FVM for solving ADE. They stated that using soft-computing techniques for predicting internal coefficients of governing differential equations results in an increase in the accuracy of numerical simulation. In this paper, multivariate adaptive regression splines (MARS) is used as a new approach in hydrology for modelling and prediction of $D_{L}$. To compare performance of MARS model with other types of soft-computing techniques, multilayer perceptron neural network (MLP) as a common type of artificial neural network is also developed. To define the most effective parameters on $D_{L}$, the Gamma test (GT) was applied. Results of GT are used to evaluate the results of MARS model as well. MARS model was developed by Friedman (1991) and has been used in some fields of water resource management even today. MARS technique has been successfully applied for predicting scour depth at downstream of spillways, river discharge forecasting, rainfallrunoff modelling, etc. (Sharda et al. 2008; Zhang and Goh 2014; Samadi et al. 2015).

\section{Materials and methods}

Initially, a review is conducted on the influence of hydraulic and geometric parameters on $D_{L}$. Then, using the Buckingham, dimensionless parameters are derived. Later, using the Gamma test, the most effective parameters on $D_{L}$ are defined. At the end, two soft-computing techniques including ANN and MARS model are developed and compared.

\subsection{Relevant parameters and dimensional analysis}

Longitudinal dispersion coefficient is proportional to properties of fluid, hydraulic conditions and river geometry (cross-sections and path line). All effective parameters can be written as the following function:

$$
D_{L}=f_{1}\left(\rho, \mu, u, u_{*}, h, w, s_{f}, s_{n}\right)
$$

where $\rho$ is fluid density; $\mu$ is dynamic viscosity; $w$ is width of cross-section; $h$ is flow-depth; $u_{*}$ is shear velocity, $s_{f}$ is longitudinal bed shape and $s_{n}$ is sinuosity. The Buckingham theory was applied as dimensional analysis approach to derive effective dimensionless parameter on $D_{L}$. Derived 
Table 1. Empirical equations for estimating longitudinal dispersion coefficient.

\begin{tabular}{lll}
\hline Sl. no. & \multicolumn{1}{c}{ Author } & \multicolumn{1}{c}{ Equation } \\
\hline 1 & Elder (1959) & $D_{L}=5.93 h u_{*}$ \\
2 & McQuivey and Keefer (1974) & $D_{L}=0.58\left(\frac{h}{u_{*}}\right)^{2} u w$ \\
3 & Fisher (1967) & $D_{L}=0.011 \frac{u^{2} w^{2}}{h u_{*}}$ \\
4 & Li et al. (1998) & $D_{L}=0.55 \frac{w u_{*}}{h^{2}}$ \\
5 & Liu (1977) & $D_{L}=0.18\left(\frac{u}{u_{*}}\right)^{0.5}\left(\frac{w}{h}\right)^{2} h u_{*}$ \\
6 & Iwasa and Aya (1991) & $D_{L}=2\left(\frac{w}{h}\right)^{1.5} h u_{*}$ \\
7 & Seo and Cheong (1998) & $D_{L}=5.92\left(\frac{u}{u_{*}}\right)^{1.43}\left(\frac{w}{h}\right)^{0.62} h u_{*}$ \\
8 & Koussis and Rodriguez-Mirasol (1998) & $D_{L}=0.6\left(\frac{w}{h}\right)^{2} h u_{*}$ \\
9 & Li et al. (1998) & $D_{L}=5.92\left(\frac{u}{u_{*}}\right)^{1.2}\left(\frac{w}{h}\right)^{1.3} h u_{*}$ \\
10 & Kashefipour and Falconer (2002) & $D_{L}=2\left(\frac{u}{u_{*}}\right)^{0.96}\left(\frac{w}{h}\right)^{1.25} h u_{*}$ \\
11 & Tavakollizadeh and Kashefipur (2007) & $D_{L}=7.428+1.775\left(\frac{u}{u_{*}}\right)^{1.752}\left(\frac{w}{h}\right)^{0.62} h u$ \\
\hline 2 & Rajeev and Dutta (2009) & $D_{L}=10.612\left(\frac{u}{u_{*}}\right)^{h u}$ \\
\hline
\end{tabular}

Table 2. Ranges of collected data related to $D_{L}$.

\begin{tabular}{lrrccr}
\hline Data range & \multicolumn{1}{c}{$\begin{array}{c}H \\
(\mathrm{~m})\end{array}$} & $\begin{array}{c}\text { U } \\
(\mathrm{m})\end{array}$ & $\begin{array}{c}U \\
(\mathrm{~m} / \mathrm{s})\end{array}$ & $\begin{array}{c}U^{*} \\
(\mathrm{~m} / \mathrm{s})\end{array}$ & $\begin{array}{c}D_{L} \\
\left(\mathrm{~m}^{2} / \mathrm{s}\right)\end{array}$ \\
\hline Min & 11.9 & 0.2 & 0.0 & 0.0 & 1.9 \\
Max & 711.2 & 19.9 & 1.7 & 0.6 & 1486.5 \\
Avg & 73.2 & 1.5 & 0.5 & 0.1 & 115.3 \\
Stdev & 106.9 & 2.3 & 0.4 & 0.1 & 218.7 \\
\hline
\end{tabular}

dimensionless parameters are shown in equation (3) (Seo and Cheong 1998),

$$
\frac{D_{L}}{h u_{*}}=f_{2}\left(\rho \frac{u h}{\mu}, \frac{u}{u_{*}}, \frac{w}{h}, s_{f}, s_{n}\right) .
$$

Flow-in nature, especially in rivers, is always turbulent. Therefore, the Reynolds number $\rho(u h / \mu)$ can be ignored and bed form and sinusitis path parameters cannot be measured clearly, as well. Therefore, their effects can be considered as flow resistant, which is seen in flow depth. Dimensionless parameters that can be clearly measured are given as below (Seo and Cheong 1998; Seo and Baek 2004):

$$
\frac{D_{L}}{h u_{*}}=f_{2}\left(\frac{u}{u_{*}}, \frac{w}{h}\right) .
$$

Developing empirical formulas and soft-computing models are based on these dimensionless parameters. Table 1 presents the most famous empirical formulas, which have been presented for $D_{L}$ calculation.

Soft-computing models are prepared based on the dataset; therefore, for this purpose, about 116 datasets related to equation (4), were used, the ranges of which are given in table 2 (Zeng and Huai 2014).

\subsection{Gamma test (GT)}

The Gamma test is used to examine the relationship between inputs and outputs in numerical datasets without the need to construct estimation model. The Gamma test is used for estimating the variance of output before modelling, even though the model is unknown. This error variance estimate presents a target mean squared error that any smooth non-linear function should attain on unseen data. Suppose we have a set of observed data represented by:

$$
\left(\left(x_{1}, \ldots, x_{M}\right), y\right)=(x, y)
$$

where vector $X=\left(x_{1}, \ldots, x_{M}\right)$ is the input, confined to a closed bounded set $C \in R^{M}$ and scalar $y$ is the corresponding output, without loss of generality. The only assumption made is that relationship of the system is in the following form:

$$
y=f\left(x_{1}, \ldots, x_{M}\right)+r
$$

where $f$ represents a smooth function and $r$ denotes an indeterminable part, which may be due to real noise lack of functional determination in assumed input/output relationship. The Gamma test is used to return a data-derived estimate for $\operatorname{Var}(r)$ without knowing the underlying function $(f)$ just directly from the data. Estimate of model's output variance called the Gamma statistic represented by $\Gamma$, cannot be accounted for by a smooth data model. The Gamma test is derived from the Delta function of input vectors:

$$
\delta_{M}(k)=\frac{1}{M} \sum_{i=1}^{M}\left|x_{N[i, k]}-x_{i}\right|^{2}
$$


where $x_{N[i, k]}$ denotes the index of $k$ th nearest tance. Thus, $\delta_{M}(k)$ is the mean square distance to $k$ th nearest neighbour. The corresponding Gamma function of output values is:

$$
\gamma_{M}(k)=\frac{1}{2 M} \sum_{i=1}^{M}\left(y_{N[i, k]}-y_{i}\right)^{2} .
$$

The Gamma test computes the mean-squared $k$ th nearest neighbour distances $\delta(k),\left(1 \leq k \leq k_{\text {Max }}\right)$ and the corresponding $\gamma(p)^{2}$. In order to compute $\Gamma$, the best line is constructed for $p$ points $\left(\delta_{M}(k)\right.$, $\left.\gamma_{M}(k)\right)$ and vertical intercept $(\Gamma)$, is returned as the gamma value. The regression line slope is also returned to show the complexity of model $f$. $V_{\text {ratio }}$ is the standardized results considering $\Gamma / \operatorname{Var}(y)$. It returns a scale invariant noise estimate, which normally lies between zero and one (Noori et al. 2009, 2015a, b).

\subsection{Artificial Neural Networks (ANNs)}

ANN is a nonlinear mathematical model that is able to simulate arbitrarily complex nonlinear processes of any systems that are in terms of inputs and outputs. In many complex mathematical problems that lead to solve complex nonlinear equations, multilayer perceptron networks are common types of ANN widely used in the research. To use MLP model, definition of transferring functions, weights and bias should be considered. Due to the nature of the problem, different activity functions in neurons can be used. An ANN may have one or more hidden layers. Figure 2 demonstrates a three-layer neural network consisting of inputs layer, hidden layer (layers) and outputs layer. As shown in figure $2, w_{i}$ is the weight and $b_{i}$ is the bias for each neuron. Weight and biases' values will be assigned progressively and corrected during training process comparing predicted outputs with neighbour to $x_{i}$, and $|\cdot|$ denotes Euclidean dis-

known outputs. Such networks are often trained using back propagation algorithm. In the present study, ANN was trained by Levenberg-Marquardt technique since this technique is more powerful and faster compared to conventional gradient descent technique.

\subsection{Multivariate adaptive regression splines (MARS)}

MARS technique is a novel approach in softcomputing that applies a series of simple linear regressions. As mentioned in the introduction section, MARS was introduced by the mathematician Friedman (1991). It is a high-precision technique for modelling systems which is based on dataset. This approach separated the computational space into sub-ranges of input variables (predicting parameters) and defined the relationship between input parameters and output variable. In other words, this technique has high ability to characterize the relationship between independent variables and dependent variable in each desired phenomenon. This process was carried out by fitting a simple regression on each input parameter for predicting the output. MARS separates the space of input parameters into various units and then fits a spline function on these units. These elements of regressions are called basic function of MARS methods. One of the main advantages of MARS method is highlighting input parameters which have prominent effect on output parameter. This method could be used for small and big datasets. A basic function that gives information about the relationship between inputs and output parameters is defined as equation (9).

$$
h_{m}(x)=\operatorname{Max}(0, C-x)
$$

or

$$
h_{m}(x)=\operatorname{Max}(0, x-C)
$$
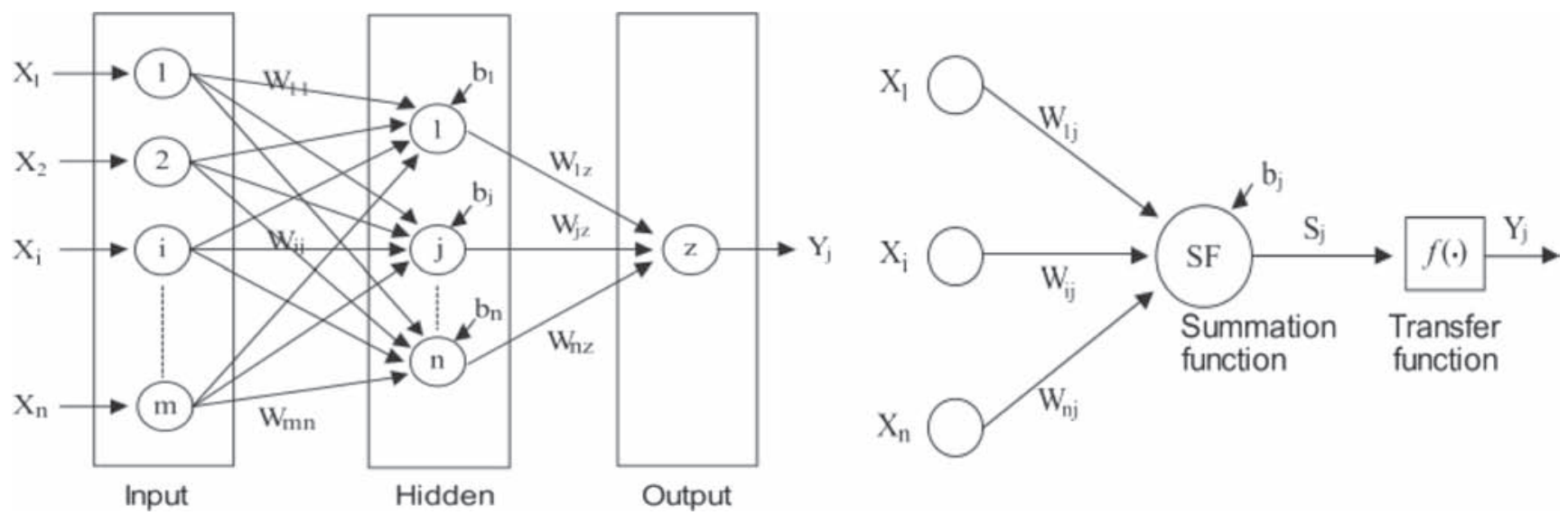

Figure 2. Sketch of three-layer ANN architecture. 
where $h$ is the basic function, $x$ is the input parameter, and $C$ is the threshold value of the independent (input) parameter of $x$. It is notable that $C$ is introduced in scientific paper via knots and is derived using the least square method. The general form of MARS is introduced as equation (10).

$$
Y=f(x)=\beta_{0}+\sum_{m=1}^{M} \beta_{m} h_{m}(x)
$$

where $Y$ is output parameters, $\beta_{0}$ is constant value, $M$ is the number of functions, $h_{m}(x)$ is $M$ th basic function and $\beta_{m}$ is the corresponding coefficient of $h_{m}(x)$. Developing MARS includes two steps. In the first step, all basic functions are prepared where overfitting can occur. Therefore, to prevent overfitting in the next step, basic functions, which are less important are pruned with generalized cross-validation (GCV) criteria that is given in equation (11).

$$
\mathrm{GCV}=\frac{\frac{1}{n} \sum_{i=1}^{n}\left(y_{i}-f\left(x_{i}\right)\right)^{2}}{\left(1-\left(\frac{C(B)}{n}\right)\right)^{2}}
$$

where $n$ denotes the number of observations and $C(B)$ denotes complexity criteria which increase by the number of basic functions (12).

$$
C(B)=(B+1)+d B .
$$

\subsection{Neural network model development}

Developing MLP model as a soft-computing technique is based on the dataset. Therefore, for this purpose, collected dataset was divided into two groups as training and testing. Data selection for ANN model training and testing process was carried out by random approach. The percentage of each group portfolio from total dataset will be determined during model preparation. Designing ANN model structure is more based on designer experience; however, recommendations of investigators who have conducted similar researches were also taken into consideration. Designing ANN model includes a type of neural network model, number of hidden layer(s), number of neurons in each layer, and defining suitable transfer function for neurons of hidden and output layers and learning algorithm.

\section{Results and discussion}

Performance of empirical formulae was assessed for predicting $D_{L}$ and is shown in figure 3. For this purpose, three standard error indices including $R^{2}$, root mean square error and mean absolute error were used. Results of error indices calculation for each empirical formula are given in table 3 . As presented in table 3, all empirical formulae have determination of correlation $<50 \%$ and this accuracy is not suitable for practical usage. As seen from table 3, the Tavakollizadeh and Kashefipur formula (2007) is the accurate among empirical formulae.

\subsection{Results of Gamma test}

In this study, to define the most important effective parameters on $D_{L}$, five scenarios were considered. In each scenario, the effect of the input variables was evaluated. In the first scenario, all input variables were used for the GT and in the next scenarios, one of the input variables was removed and again the GT was analyzed. Result of each scenario is given in table 4. The GT parameters including gamma, gradient, standard error and $V_{\text {ratio }}$ were chosen as criteria for defining the most important parameters. Scenarios, which had minimum value for the GT parameters showed the most influential input parameters on output parameter. Variation of $V_{\text {ratio }}$ is between 0 and 1 . The point notable here is that the more this factor is close to zero, the more accurate the related scenario can predict the output regarding related input parameters.

Reviewing table 4 , it is realized that scenario number (1) which involves all input variables has minimum value for the GT parameters. Table 4 shows that removing $U / u^{*}$ causes significant decrease in the Gamma value; therefore, it was found that these parameters are the most important parameters on $D_{L}$ modelling. Variation of gamma for dataset based on all input variables is given in figure 4 . Figure 4 shows that the gamma curves are almost flat after point 100 . It means that for modelling discharge coefficient regrading collected dataset, qualification of 100 dataset $(85 \%$ of all datasets) is sufficient.

\subsection{Results of ANN model development}

Developing ANN model includes choosing dataset for training and defining portfolio of training and testing groups from total dataset, choosing the number of hidden layer(s), number of neurons in each hidden layer(s), choosing transfer function for neurons and finally choosing learning algorithm. To provide MLP model for predicting $D_{L}$, utilities of Matlab software were used. Table 5 presents a summary of trial-and-error process for preparing ANN model. To develop ANN model, $70 \%$ of collected dataset was considered for training, $15 \%$ for validation and the rest (15\%) for testing. Validation of dataset was considered to avoid overlearning. As can be seen from table 5 , ANN model, which has 10 

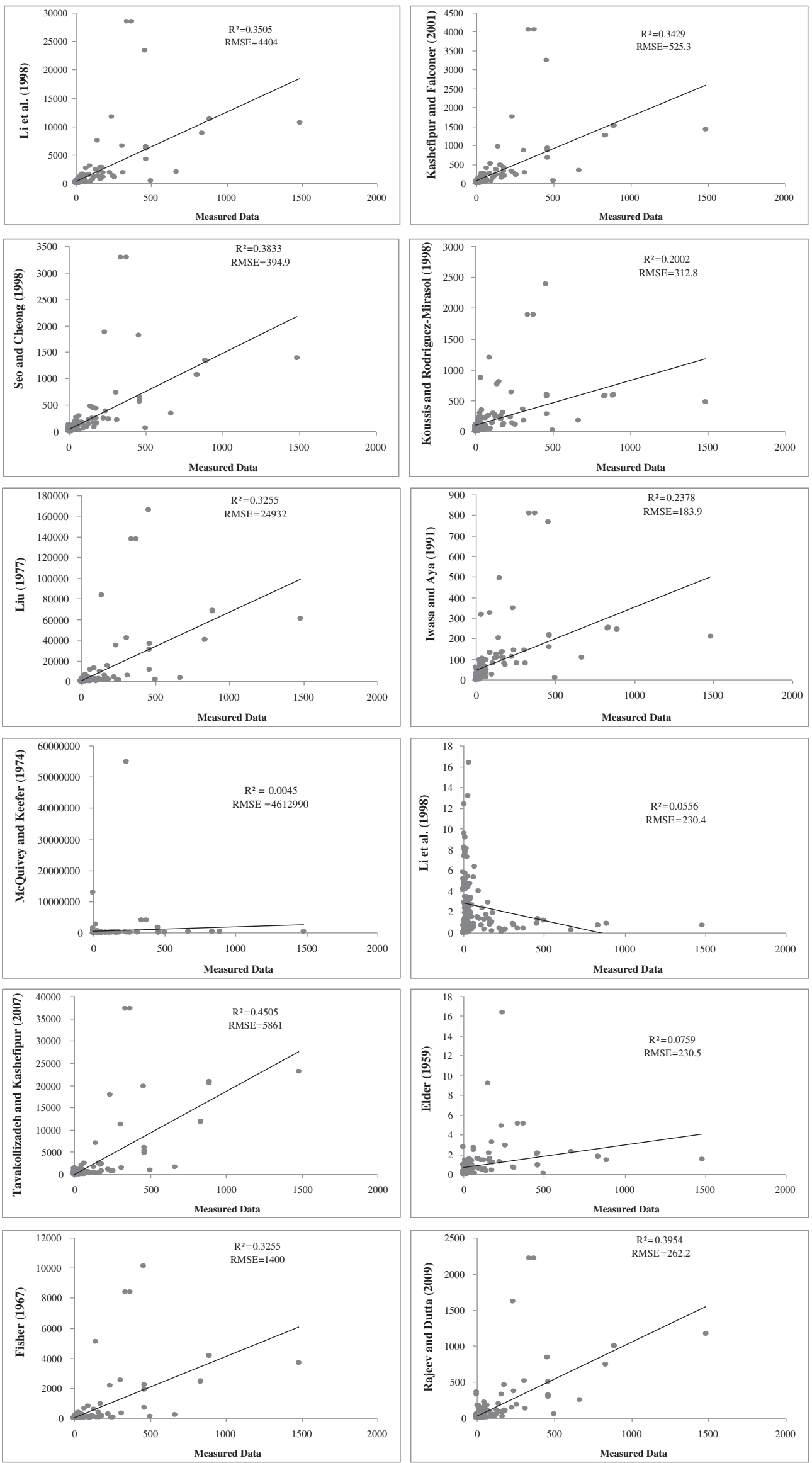

Figure 3. Performance of empirical formulas to calculate $D_{L}$. 
Table 3. Performance of empirical formulae to calculate $D_{L}$.

\begin{tabular}{lcrr}
\hline Equation & $R^{2}$ & RMSE & MAPE \\
\hline Elder (1959) & 0.076 & 230.05 & 0.96 \\
McQuivey and Keefer (1974) & 0.004 & 4612990 & 465028 \\
Fisher (1967) & 0.326 & 1301.03 & 3.25 \\
Li et al. (1998) & 0.056 & 230.38 & 0.87 \\
Liu (1977) & 0.326 & 24932.62 & 60.58 \\
Iwasa and Aya (1991) & 0.238 & 183.83 & 1.25 \\
Seo and Cheong (1998) & 0.383 & 394.92 & 4.40 \\
Koussis and Rodriguez-Mirasol (1998) & 0.200 & 312.85 & 2.81 \\
Li et al. (1998) & 0.351 & 4404.65 & 24.75 \\
Kashefipur and Falconer (2001) & 0.343 & 525.30 & 3.51 \\
Tavakollizadeh and Kashefipur (2007) & 0.451 & 5861.86 & 42.84 \\
Rajeev and Dutta (2009) & 0.395 & 262.20 & 8.95 \\
\hline
\end{tabular}

Table 4. Results of Gamma test analysis in the absence of one variable.

\begin{tabular}{llccccc}
\hline Row & \multicolumn{1}{c}{ Inputs } & Output & Gammas & Gradient & Standard error & $V_{\text {ratio }}$ \\
\hline 1 & $h, u^{*}, U / u^{*}, W / h$ & $D_{L}$ & 0.0935 & 0.0773 & 0.0339 & 0.3740 \\
2 & $u^{*}, U / u^{*}, W / h$ & $D_{L}$ & 0.0929 & 0.1272 & 0.0302 & 0.3714 \\
3 & $h, U / u^{*}, W / h$ & $D_{L}$ & 0.0957 & 0.1477 & 0.0375 & 0.3828 \\
4 & $h, u^{*}, W / h$ & $D_{L}$ & 0.0028 & 0.4068 & 0.0514 & 0.0113 \\
5 & $h, u^{*}, U / u^{*}$ & $D_{L}$ & 0.0753 & 0.1716 & 0.0565 & 0.3011 \\
\hline
\end{tabular}

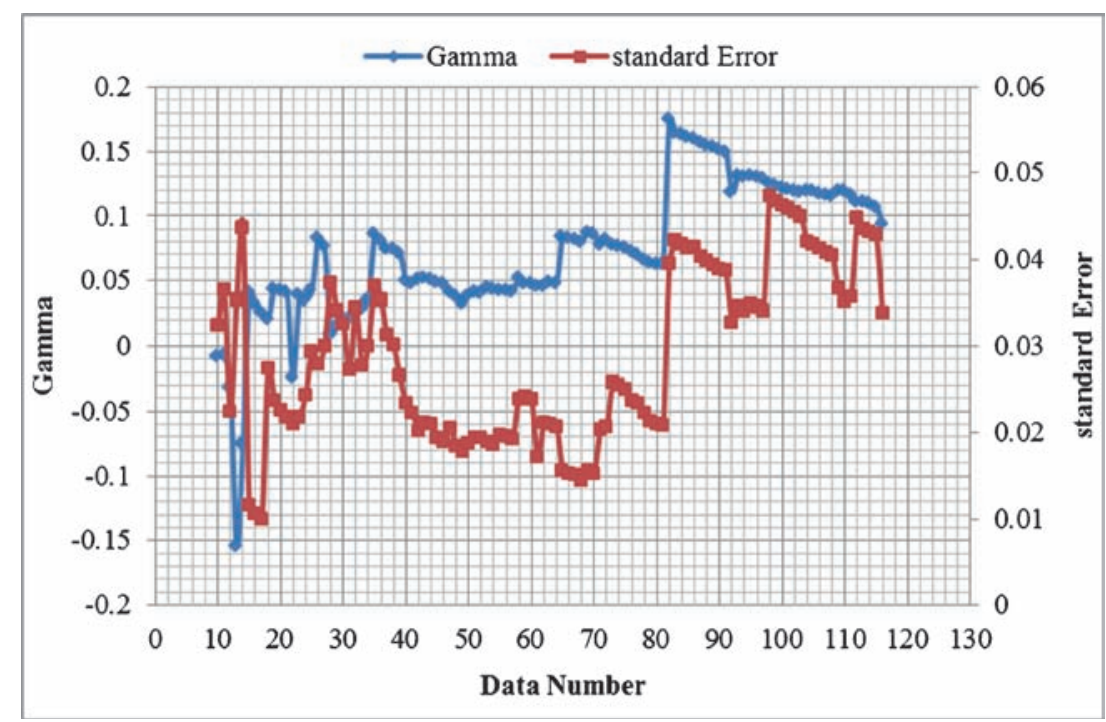

Figure 4. Variation of the Gamma test and standard error with unique data points.

neurons with logsig as transfer function in the first layer, is accurate and adding the number of hidden layer(s) and neurons in the layer has no significant effect on increasing model performance.

Performance of ANN model during stages of model preparation (training, validation and testing) is shown in figures $5-7$. In figures $5-7$, results of ANN model were plotted against measured data. In addition, to understand the performance of ANN model for training and testing data, error distribution was also plotted. Moreover, to define error concentration, error histogram was plotted.
As can be seen from the histogram of error, density of error is aggregated around zero. In overall, the performance of MLP model is more suitable especially when compared to empirical formulae.

\subsection{Results of MARS model development}

Similar to other types of soft-computing models, developing MARS model is based on the dataset. For this purpose, collected dataset (equation 4) was randomly divided into two groups of training and testing. Eighty five percent of the total data 
Table 5. Performance and summary of ANN model during development stage.

\begin{tabular}{lccccccccc}
\hline Row & N-H-L & F-HL\&TF & S-HL\&TF & $R^{2}$ & RMSE & MAE & $R^{2}$ & RMSE & MAE \\
\hline 1 & 1 & 5-tansig & - & 0.55 & 0.286 & 0.0 & 0.77 & 0.04 & 0.015 \\
2 & 1 & 8-tansig & - & 0.83 & 0.084 & 0.03 & 0.75 & 0.03 & 0.018 \\
3 & 1 & 5-logsig & - & 0.86 & 0.04 & 0.016 & 0.85 & 0.05 & 0.012 \\
4 & 1 & 8-logsig & - & 0.83 & 0.046 & 0.024 & 0.72 & 0.052 & 0.016 \\
5 & 1 & 10-logsig & - & 0.93 & 0.044 & 0.015 & 0.88 & 0.003 & 0.054 \\
6 & 2 & 9-logsig & 5-tansig & 0.93 & 0.053 & 0.028 & 0.86 & 0.060 & 0.012 \\
7 & 2 & 12-tansig & 5-tansig & 0.95 & 0.063 & 0.035 & 0.95 & 0.068 & 0.015 \\
\hline
\end{tabular}
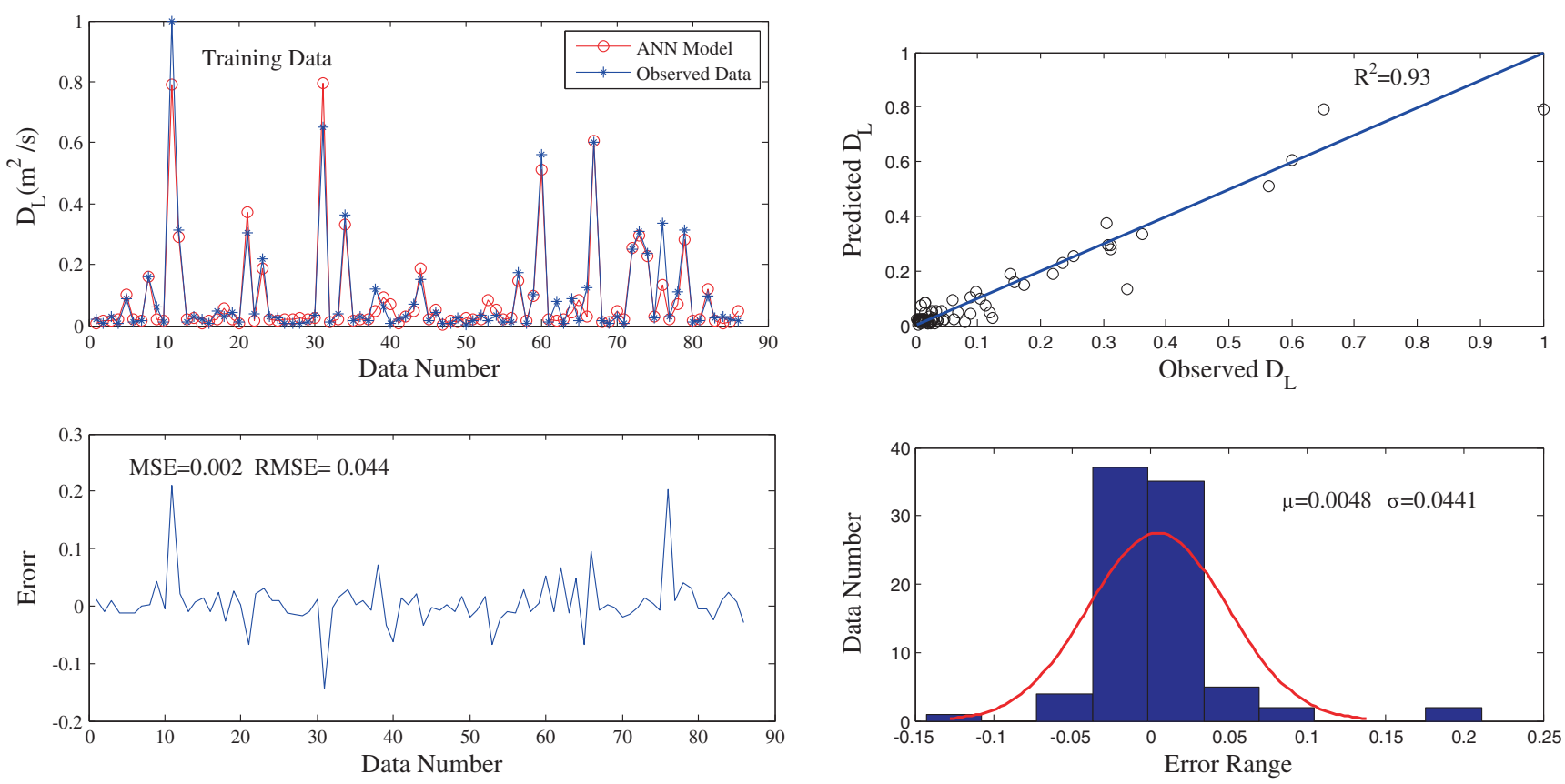

Figure 5. Performance of MLP model during training stage.
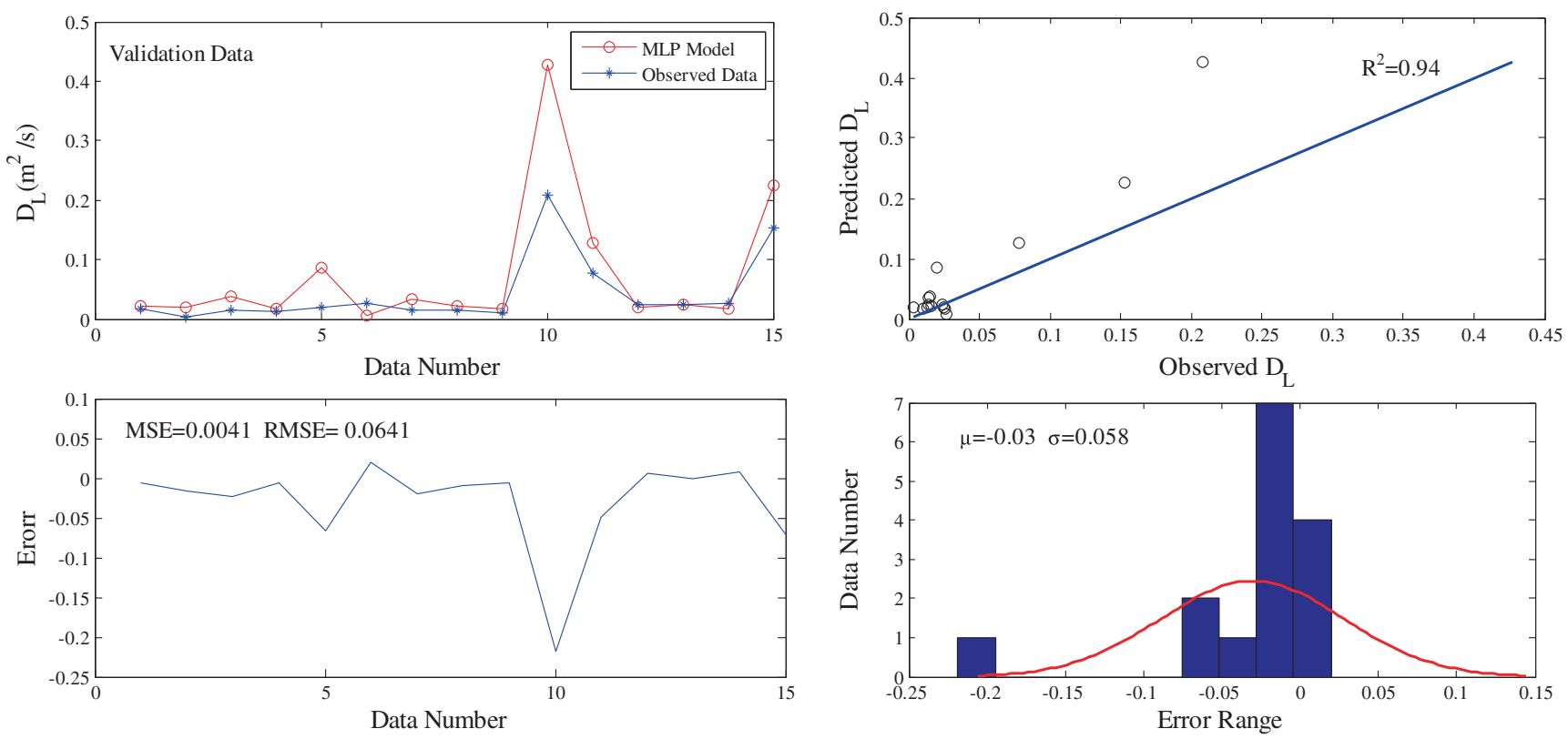

Figure 6. Performance of MLP model during validation stage. 

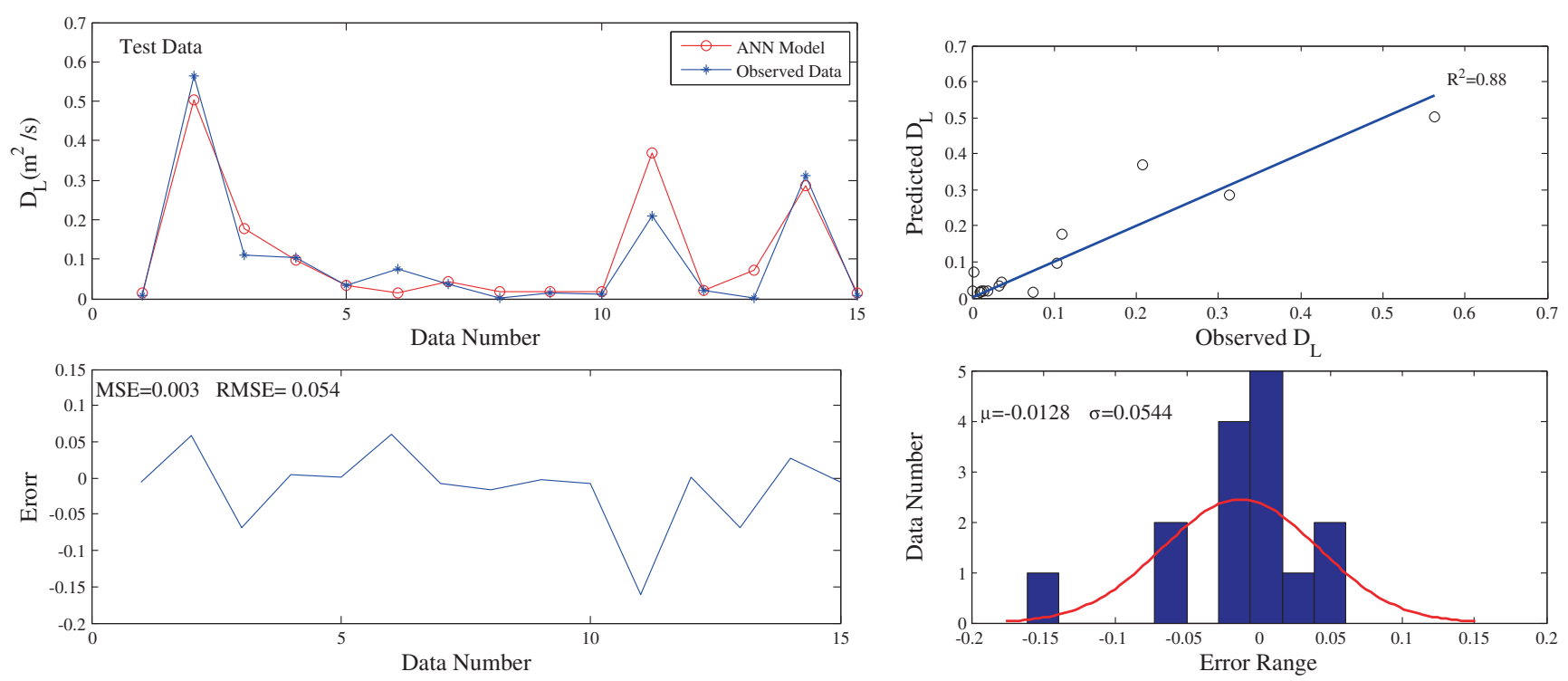

Figure 7. Performance of MLP model during testing stage.

Table 6. The Basic function and related coefficients of MARS model.

\begin{tabular}{llr}
\hline Basic function & \multicolumn{1}{c}{ Equation } & Coefficient \\
\hline$h_{1}(\mathrm{x})$ & $\mathrm{BF} 1=\max \left(0,0.63651-u / u^{*}\right)$ & 2.3410 \\
$h_{2}(\mathrm{x})$ & $\mathrm{BF} 2=\max (0, h-0.14655)$ & -9.7300 \\
$h_{3}(\mathrm{x})$ & $\mathrm{BF} 3=\max (0,0.14655-h)$ & 16.4736 \\
$h_{4}(\mathrm{x})$ & $\mathrm{BF} 4=\mathrm{BF} 2 * \max \left(0, u / u^{*}-0.7162\right)$ & 292.4690 \\
$h_{5}(\mathrm{x})$ & $\mathrm{BF} 5=\mathrm{BF} 2 * \max \left(0,0.7162-u / u^{*}\right)$ & 100.7754 \\
$h_{6}(\mathrm{x})$ & $\mathrm{BF} 6=\mathrm{BF} 1 * \max (0,0.13793-h)$ & -102.7943 \\
$h_{7}(\mathrm{x})$ & $\mathrm{BF} 7=\max (0,0.0974-w)$ & 2.8615 \\
$h_{8}(\mathrm{x})$ & $\mathrm{BF} 8=\max (0, w-0.24124)$ & 1.4645 \\
$h_{9}(\mathrm{x})$ & $\mathrm{BF} 9=\max (0,0.24124-w)$ & -14.3571 \\
$h_{10}(\mathrm{x})$ & $\mathrm{BF} 10=\max \left(0, u / u^{*}-0.7162\right) * \max (0, w-0.24124)$ & 37.6451 \\
$h_{11}(\mathrm{x})$ & $\mathrm{BF} 11=\max \left(0, u / u^{*}-0.7162\right) * \max (0,0.24124-w)$ & -0.3316 \\
$h_{12}(\mathrm{x})$ & $\mathrm{BF} 12=\max \left(0, u / u^{*}-0.44949\right)$ & 2.0688 \\
$h_{13}(\mathrm{x})$ & $\mathrm{BF} 13=\mathrm{BF} 1 * \max (0, w-0.065808)$ & -105.5226 \\
$h_{14}(\mathrm{x})$ & $\mathrm{BF} 14=\mathrm{BF} 1 * \max (0, h-0.1141)$ & 103.4887 \\
$h_{15}(\mathrm{x})$ & $\mathrm{BF} 15=\mathrm{BF} 1 * \max (0,0.1141-h)$ & 98.6693 \\
$h_{16}(\mathrm{x})$ & $\mathrm{BF} 16=\mathrm{BF} 9 * \max (0, h-0.03144)$ & -99.1440 \\
$h_{17}(\mathrm{x})$ & $\mathrm{BF} 17=\mathrm{BF} 9 * \max (0,0.03144-h)$ & -118.6282 \\
$h_{18}(\mathrm{x})$ & $\mathrm{BF} 18=\mathrm{BF} 3 * \max (0, w-0.067996)$ & 97.9521 \\
$h_{19}(\mathrm{x})$ & $\mathrm{BF} 19=\mathrm{BF} 3 * \max (0,0.067996-w)$ & \\
\hline & &
\end{tabular}

was considered for training and the rest (15\%) for testing group. During MARS model preparation, in the first step, 30 basic functions were considered and in the second step (pruning step), 11 basic functions were pruned and finally, optimal MARS model with 19 basic functions was derived. Pruning criteria, which were introduced with GVC parameter in the development of MARS model, were derived equal to 0.0063. As mentioned in the section of a review on MARS model, each basic function has coefficient and constant, which was adjusted in MARS model development process derived using the least square method. The general form of obtained MARS model is given in equation (13). The extended form of MARS model is given in table 6 .

$$
D_{L}=0.560+\sum_{M=1}^{19} \beta_{m} h_{m}(x) .
$$

Equation (13) can be used for longitudinal dispersion coefficient in rivers. Considering table 6 , $u / u^{*}$ and $h$ have appeared in almost all basic functions. It means that these two parameters, compared to other parameters, are more effective on $D_{L}$. This result from MARS model upholds the 


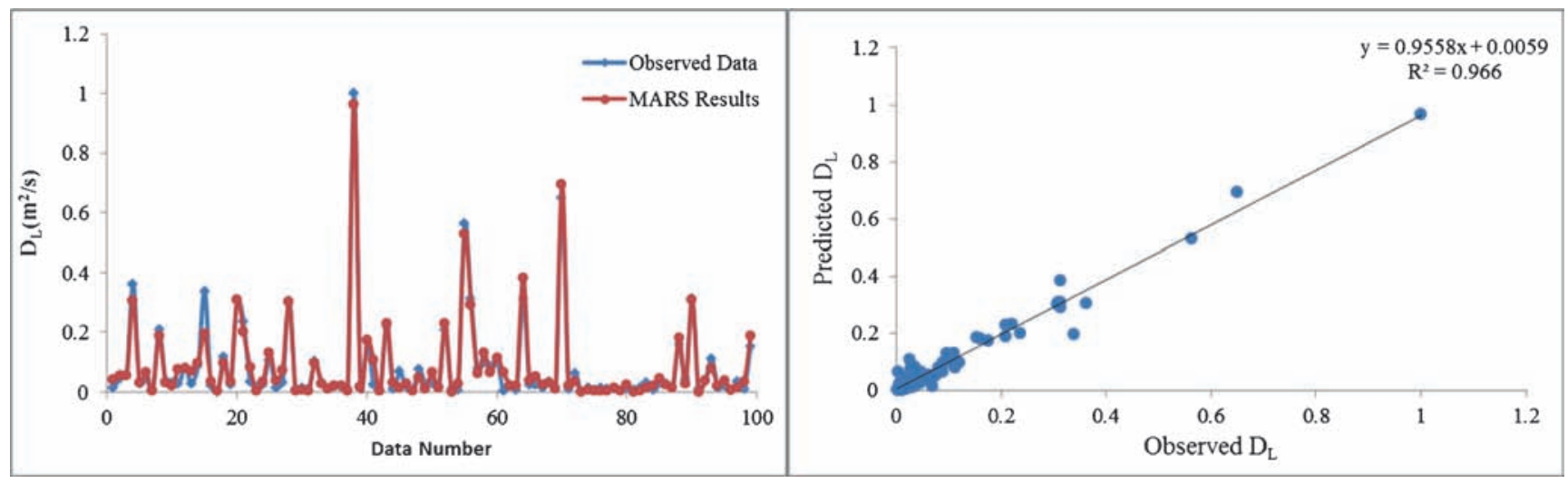

Figure 8. Performance of MARS model during training stage.

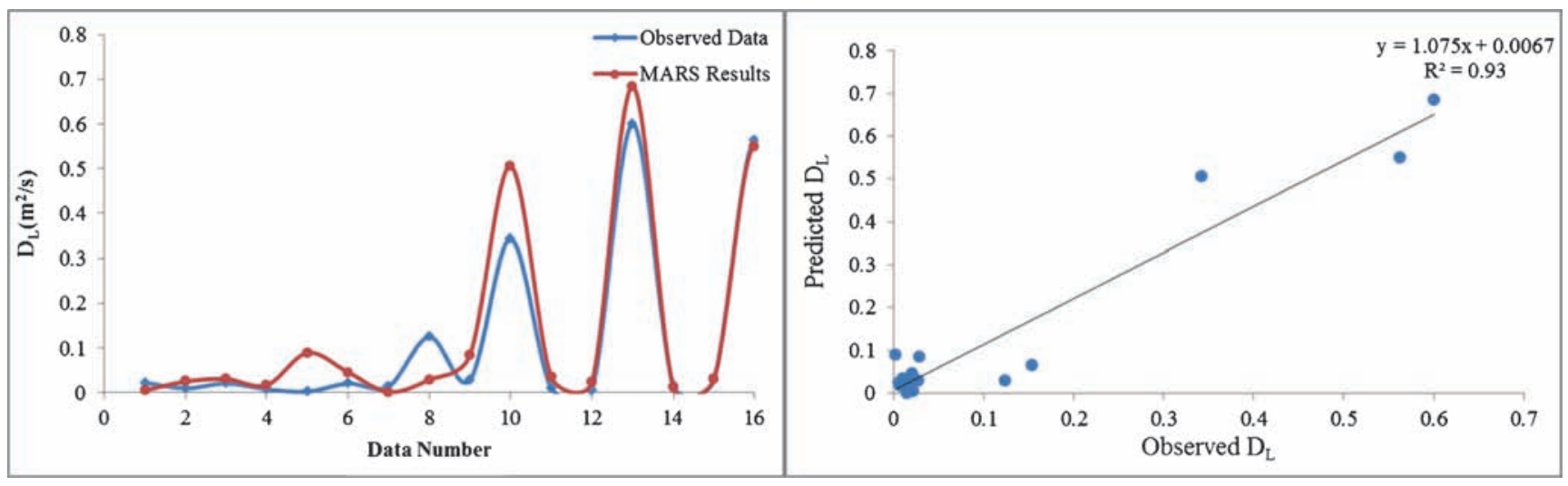

Figure 9. Performance of MARS model during testing stage.

results of the GT model. Moreover, by considering the results of assessing performance of empirical formula and MARS model results, it is clear that empirical formula, which has more weight over these formula such as Tavakollizadeh and Kashefipur (2007), is more accurate compared to others. Performance of MARS model during development process (training and testing stages) is given in figures 8 and 9 . As can be seen from figures 8 and 9 , it is found that performance of MARS model for prediction of $D_{L}$ is more suitable. Another advantage of developed MARS model is in terms of utility in programming the results of MARS model for developing other river pollution packages.

\section{Conclusion}

River pollution has become the most important issue in environmental engineering. Dispersion coefficient $\left(D_{L}\right)$ is one of the main parameters in river water quality studies. Recently, by advances in soft-computing techniques in hydrology engineering, investigators attempt to use these models for predicting $D_{L}$. Among the soft-computing techniques, multi-layer perceptron neural network as primary model has been used in most areas of engineering and science, especially in hydrology engineering. Researchers have reported that this model is a black box and does not give any information about internal model process. Recently, by proposing the newest types of soft-computing techniques such as multivariate adaptive regression splines (MARS) in addition to proposing highperformance techniques for predicting engineering phenomena, this model gives full information to designers and readers. In other words, the internal process of MARS model is clear for readers and users. In this paper, developing MARS model for predicting $D_{L}$ was considered. Results of this research show that MARS model has high performance for predicting $D_{L}$ and gives full information about internal model process to the readers. Users can easily prepare MARS model for other researches.

\section{References}

Atkinson T and Davis P 2000 Longitudinal dispersion in natural channels: 1. Experimental results from the River Severn, UK; Hydrol. Earth Syst. Sci. 4(3) 345-353. 
Azamathulla H and Ghani A 2011 Genetic programming for predicting longitudinal dispersion coefficients in streams; Water Resour. Manag. 25(6) 1537-1544.

Azamathulla H M and Wu F-C 2011 Support vector machine approach for longitudinal dispersion coefficients in natural streams; Appl. Soft Comput. 11(2) 2902-2905.

Benedini M and Tsakiris G 2013 Water quality modelling for rivers and streams; Springer Science \& Business Media.

Chao X, Jia Y, Shields F D, Wang S S and Cooper C M 2007 Numerical modeling of water quality and sediment related processes; Ecol. Modelling 201(3) 385-397.

Davis P and Atkinson T 2000 Longitudinal dispersion in natural channels: 3 . An aggregated dead zone model applied to the River Severn, UK; Hydrol. Earth Syst. Sci. 4(3) $373-381$.

Davis P, Atkinson T and Wigley T 2000 Longitudinal dispersion in natural channels: 2 . The roles of shear flow dispersion and dead zones in the River Severn, UK; Hydrol. Earth Syst. Sci. 4(3) 355-371.

Disley T, Gharabaghi B, Mahboubi A A and McBean E A 2015 Predictive equation for longitudinal dispersion coefficient; Hydrol. Process. 29(2) 161-172.

Elder J W 1959 The dispersion of a marked fluid in turbulent shearflow; J. Fluid Mech. 5(4) 544-560.

El Kadi Abderrezzak K, Ata R and Zaoui F 2015 Onedimensional numerical modelling of solute transport in streams: The role of longitudinal dispersion coefficient; J. Hydrol. 527 978-989.

Fisher B H 1967 The mechanics of dispersion in natural streams; J. Hydraul. Div. 93(6) 187-216.

Friedman J H 1991 Multivariate adaptive regression splines; The Annals of Statistics 19 1-67.

Iwasa Y and Aya S 1991 Predicting longitudinal dispersion coefficient in open channel flows; In: Proceedings of International Symposium on Environmental Hydraulics, Hong Kong, pp. 505-510.

Ji Z-G 2008 Hydrodynamics and water quality: Modeling rivers, lakes, and estuaries; John Wiley \& Sons.

Kashefipour S M and Falconer R A 2002 Longitudinal dispersion coefficients in natural channels; Water Res. 36(6) 1596-1608, doi: 10.1016/S0043-1354(01)00351-7.

Koussis A D and Rodriguez-Mirasol J 1998 Hydraulic estimation of dispersion coefficient for streams; J. Hydraul. Eng. 124 317-320.

Leta O, Shrestha N, de Fraine B, van Griensven A and Bauwens W 2014 Integrated water quality modelling of the river Zenne (Belgium) using open MI; In: Advances in Hydroinformatics (eds) P Gourbesville, J Cunge and G Caignaert, Springer Singapore, pp. 259-274.

Li Z H, Huang J and Li J 1998 Preliminary study on longitudinal dispersion coefficient for the gorges reservoir; In: Proceedings of the seventh International Symposium Environmental Hydraulics, 16-18 December, Hong Kong, China.

Liu H 1977 Predicting dispersion coefficient of stream; $J$. Environ. Eng. Div. 103(1) 59-69.

McQuivey R S and Keefer T N 1974 Simple method for predicting dispersion in streams; J. Environ. Eng. Div. 100(4) 997-1011.

Najafzadeh M and Sattar A A 2015 Neuro-Fuzzy GMDH approach to predict longitudinal dispersion in water networks; Water Resour. Manag. 29(7) 2205-2219.

Noori R, Karbassi A, Farokhnia A and Dehghani M 2009 Predicting the longitudinal dispersion coefficient using support vector machine and adaptive Neuro-Fuzzy inference system techniques; Environ. Eng. Sci. 26(10) $1503-1510$.
Noori R, Deng Z, Kiaghadi A and Kachoosangi F T 2015a How reliable are ANN, ANFIS, and SVM techniques for predicting longitudinal dispersion coefficient in natural rivers? J. Hydraul. Eng. 04015039.

Noori R, Yeh H-D, Abbasi M, Kachoosangi $F$ T and Moazami S 2015b Uncertainty analysis of support vector machine for online prediction of five-day biochemical oxygen demand; J. Hydrol. 527 833-843.

Parsaie A and Haghiabi A 2015a Computational modeling of pollution transmission in rivers; Appl. Water Sci., pp. 1-10, doi: 10.1007/s13201-015-0319-6.

Parsaie A and Haghiabi A 2015b The effect of predicting discharge coefficient by neural network on increasing the numerical modeling accuracy of flow over side Weir; Water Resour. Manag. 29(4) 973-985.

Parsaie A and Haghiabi A 2015c Predicting the longitudinal dispersion coefficient by radial basis function neural network; Modeling Earth Systems and Environment 1(4) 1-8.

Parsaie A and Haghiabi A H 2015d Calculating the longitudinal dispersion coefficient in river. Case study: Severn River, UK; Int. J. Sci. Res. Environ. Sci. 3(5) 199-207.

Rajeev R S and Dutta S 2009 Prediction of longitudinal dispersion coefficients in natural rivers using genetic algorithm; Hydrol. Res. 40(6) 544-552.

Riahi-Madvar H, Ayyoubzadeh S A, Khadangi $\mathrm{E}$ and Ebadzadeh M M 2009 An expert system for predicting longitudinal dispersion coefficient in natural streams by using ANFIS; Expert Systems with Applications 36(4) 8589-8596.

Samadi M, Jabbari E, Azamathulla H and Mojallal M 2015 Estimation of scour depth below free overfall spillways using multivariate adaptive regression splines and artificial neural networks; Engineering Applications of Computational Fluid Mechanics 9(1) 291-300.

Sattar A M A and Gharabaghi B 2015 Gene expression models for prediction of longitudinal dispersion coefficient in streams; J. Hydrol. 524 587-596.

Seo I and Baek K 2004 Estimation of the longitudinal dispersion coefficient using the velocity profile in natural streams; J. Hydraul. Eng. 130(3) 227-236.

Seo I and Cheong T 1998 Predicting longitudinal dispersion coefficient in natural streams; J. Hydraul. Eng. 124(1) 25-32.

Sharda V, Prasher S, Patel R, Ojasvi P and Prakash C 2008 Performance of multivariate adaptive regression splines (MARS) in predicting runoff in mid-Himalayan micro-watersheds with limited data/Performances de régressions par splines multiples et adaptives (MARS) pour la prévision d'écoulement au sein de micro-bassins versants Himalayens d'altitudes intermédiaires avec peu de données; Hydrol. Sci. J. 53(6) 1165-1175.

Stamou A I 1992 Improving the numerical modeling of river water quality by using high order difference schemes; Water Res. 26(12) 1563-1570.

Tavakollizadeh A and Kashefipur S M 2007 Effects of dispersion coefficient on quality modeling of surface waters; In: Proceedings of the sixth International Symposium on River Engineering, Ahwaz, Iran, 16-18 October 2007, pp. 67-78.

Wang Z-Y, Lee J W and Melching C 2015 Water Quality Management. River Dynamics and Integrated River Management; Springer Berlin-Heidelberg, pp. 555-631.

Zeng Y and Huai W 2014 Estimation of longitudinal dispersion coefficient in rivers; J. Hydroenviron. Res. 8(1) 2-8. Zhang W and Goh A T C 2014 Multivariate adaptive regression splines and neural network models for prediction of pile drivability; Geosci. Frontiers 7(1) 45-52. 